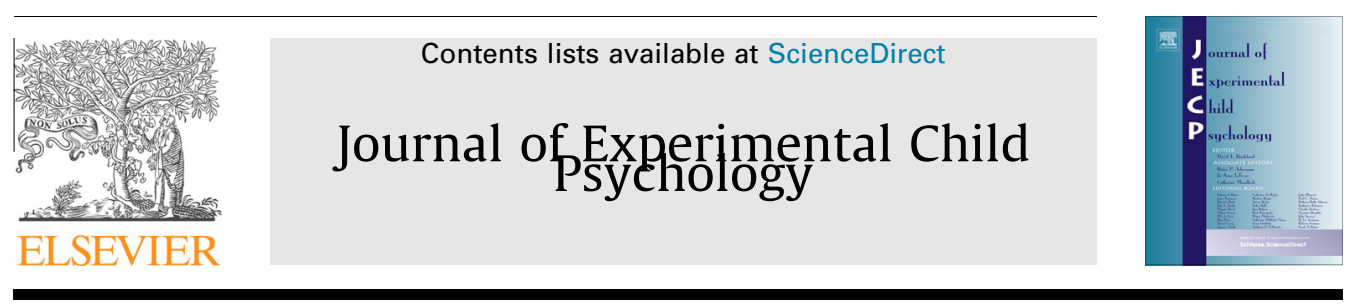

Editorial

\title{
Emerging themes in the development of prospective memory during childhood
}

\section{Introduction}

Six years ago, Kvavilashvili, Kyle, and Messer (2008) called for more research in the area of children's prospective memory (PM), defined as the ability to remember to carry out delayed intentions (Einstein \& McDaniel, 1990). At that time, the literature on PM in children was scant, although a few well-developed paradigms were available to measure PM in preschool-age children (Kvavilashvili, Messer, \& Ebdon, 2001) and older children during middle childhood (Kerns, 2000). Although there is still much work to be done, the last few years have seen a steep rise in the number of studies on the topic of PM during childhood examining children as young as 2 years using a wide variety of time- and event-based PM paradigms. This recent increase in research activity in children's $\mathrm{PM}$ was reflected in the high number of initial submissions for this special issue (20 manuscripts). The current special issue on the development of PM during childhood offers an overview of this burgeoning area of research, studying children from toddlerhood to adolescence, who are typically and atypically developing, using a wide variety of methods, including naturalistic tasks, experimental tasks, and parent report measures. In what follows, we first discuss the four sections of this special issue: PM research during early childhood, PM and episodic future thinking, PM in clinical populations, and PM during adolescence. We then highlight some emerging themes in this collection of articles that cut across these sections and highlight the contribution such topics will make to the field of PM.

\section{PM during early childhood}

The first five articles of this special issue examine the development of PM as well as the factors that influence PM during the preschool years, including the independence between PM and retrospective memory and the development of PM using a quasi-naturalistic task (Walsh, Martin, \& Courage, 2014), the negative impact of carrying out a PM task on ongoing task performance supporting the idea that controlled processes might be necessary in PM even during the preschool years (Leigh \& Marcovitch, 2014), the strong effect of an inherently motivating intention compared with a less motivating intention and the differential role that theory of mind and executive functions play in low- and high-incentive PM tasks (Causey \& Bjorklund, 2014), the role of executive function in PM showing that inhibition, in particular, fully mediates the age-related improvements in PM in 4- and 5-year-old children (Mahy, Moses, \& Kliegel, 2014), and the impressive high accuracy of 5-year-old children's predictions of their PM performance in contrast to their poor accuracy in predicting their performance on a retrospective memory task (Kvavilashvili \& Ford, 2014). All of these articles highlight understudied aspects of young children's PM development and provide results that contribute to conceptual and/or theoretical 
aspects of the development of children's PM. Furthermore, these studies all use different PM tasks that encapsulate more experimental contexts (multiple trials within a task where the action is rather arbitrary) as well as more naturalistic contexts (single PM trial where the action is a meaningful realworld action).

\section{PM and episodic future thinking}

Episodic future thinking and planning have long been suggested to play a role in children's PM (see Atance \& Jackson, 2009; Mackinlay, Kliegel, \& Mäntylä, 2009), but very little research has provided empirical support for a relation in very young children. Both articles in this section provide evidence that young children's episodic future thinking and planning ability (particularly representing a goal and a subgoal) may contribute to PM. Whereas Nigro, Brandimonte, Cicogna, and Cosenza (2014) examined the relation between 4- and 7-year-old children's performance on a PM task and that on an episodic future thinking task and found relations after controlling for age and retrospective memory, Prabhakar and Hudson (2014) examined precursors to PM, namely the ability to construct future scenarios to accomplish goals, and found developmental changes between 3- and 4-year-olds in the ability to accomplish temporally ordered goals with high working memory demands and a complex goal structure. Both studies suggest that there are important developmental changes in episodic future thinking and planning that may contribute to successful PM.

\section{PM in clinical populations}

Deficits in PM ability are often seen in children with autism spectrum disorders (e.g., Altgassen, Schmitz-Hübsch, \& Kliegel, 2010; Altgassen, Williams, Bölte, \& Kliegel, 2009; Brandimonte, Filippello, Coluccia, Altgassen, \& Kliegel, 2011; Williams, Boucher, Lind, \& Jarrold, 2012) and attention deficit disorders (e.g., Brandimonte et al., 2011; Kerns \& Price, 2001; Kliegel, Ropeter, \& Mackinlay, 2006). The two articles in the current issue use novel PM paradigms: the "Virtual Week" computerized board game to examine PM in children with autism (Henry et al., 2014) and the Cyber Cruiser-II, a computerized game in which children must guide a spaceship through outer space to examine PM in children with attention deficit/hyperactivity disorder (ADHD) (Talbot \& Kerns, 2014). Both articles document deficits in PM in these populations, but with important caveats. In 8- to 12-year-old children with autism, time-based PM performance was worse compared with controls, but no differences were shown in event-based PM. Similarly, 8- to 13-year-old children with ADHD performed worse on both time- and event-based PM, but ADHD symptomology was related to performance only on timebased PM and not on event-based PM. These studies both suggest that time-based PM is impaired in children with autism and ADHD, whereas event-based PM seems to impair only children with ADHD. In addition, both studies demonstrated that PM performance in the laboratory is related to functional outcomes in daily life measured by parent reports, supporting the ecological validity of these measures.

\section{PM during adolescence}

PM, like several other cognitive abilities that rely on prefrontal functioning, continues to develop into adolescence (e.g., Shum, Cross, Ford, \& Ownsworth, 2008; Wang, Kliegel, Yang, \& Liu, 2006). Altgassen, Vetter, Phillips, Akgun, and Kliegel (2014) show that both theory of mind and task switching, two abilities that rely on prefrontal cortex, predict PM into adolescence. These abilities have been shown to predict PM in much younger children (Ford, Driscoll, Shum, \& Macaulay, 2012; Mahy, Moses, \& Kliegel, 2014), so this is an important finding illustrating that executive and social abilities continue to contribute to PM into adolescence. Furthermore, Robey, Buckingham-Howes, Salmeron, Black, and Riggins (2014) examine the impact of prenatal drug exposure on PM abilities and brain structures related to PM. There was no effect of drug exposure on PM during mid-adolescence (around 15.5 years), although cortical thickness in frontal and parietal regions and volume of subcortical regions (i.e., putamen and hippocampus) were related to PM performance. These studies, taken together, suggest that frontal brain regions support important abilities that affect PM into adolescence, 
and PM during adolescence may be spared from the generally harmful effects of prenatal drug exposure.

\section{Emerging themes}

Beyond these four sections, several themes cut across the entire collection of articles in this special issue. We describe the following themes and their importance to the advancement of the field of PM: (a) the wide range of laboratory paradigms available for the measurement of PM, (b) a trend toward using more naturalistic measures of PM, (c) the use of reminder cues in PM measures, (d) executive functions as mechanisms of PM development, (e) the relation (or lack thereof) between PM and retrospective memory processes, and (f) testing theoretical models of PM in developmental samples.

First, this special issue nicely documents the wide range of laboratory paradigms that can be used with children from 3 years of age into the adolescent years. Walsh and colleagues' (2014) Shopping Trip Task requires young children to remember to buy certain items when they see particular stores in a slideshow; this task not only approximates children's daily experiences but also adds interest while maintaining the multiple-trial structure of many laboratory PM tasks. With 4- and 5-year-old children, Mahy and colleagues (2014) adapted the standard card-naming PM task (Kvavilashvili et al., 2001) into a card-sorting task that allows for the manipulation of ongoing task difficulty. Children were asked to help family members sort their household items into size categories of small or large either by matching size or by matching opposite size. This allowed for a better measure of ongoing task difficulty compared with card-naming tasks in which children perform very well (see Kvavilashvili et al., 2001; Mahy \& Moses, 2011). Finally, with older children (8-13 years), the CyberCruiser-II was used successfully to measure time-based PM in both typically developing children and children with ADHD (Kerns, 2000; Kerns \& Price, 2001). Children needed to navigate a spaceship through outer space and around various obstacles but also needed to remember to refuel its tank (see also Kliegel et al., 2013, for a recent adaptation of the Cruiser paradigm to measure event-based PM in school-age children and allowing the manipulation of core variables of PM models). These three PM paradigms represent a trend in the field in the development of new and creative laboratory-based PM tasks that are enjoyable for children and offer multiple trials of the PM task, which increases statistical power. Interestingly, although multiple trials in PM tasks may be less naturalistic given that daily experiences often allow only a single opportunity to realize one's intention, they offer the distinct opportunity to measure the reliability of PM across time, which may be especially critical when one considers that monitoring processes may be inconsistent across time and become more strategic over development (see Ceci \& Bronfenbrenner, 1985; Mackinlay et al., 2009; Voigt, Aberle, Schonfeld, \& Kliegel, 2011). Thus, one additional benefit of multiple PM trials is the ability to examine PM performance over time to examine its stability or malleability from trial to trial.

This issue also highlights a trend toward more naturalistic tasks that often involve a single PM trial that mimics PM tasks in children's daily lives. These tasks include children remembering to find an egg or a sticker that was hidden during a previous session (Walsh et al., 2014), reminding the experimenter to get a sticker or to flip over a testing room sign (Causey \& Bjorklund, 2014), reminding the experimenter to give a cellphone back to a colleague (Nigro et al., 2014), and having teenagers perform an action (e.g., writing down a name of a teacher) when the experimenter snaps his or her fingers (Robey et al., 2014). These single-trial tasks offer a close approximation to many daily PM tasks where there is only one single opportunity to carry out an intention in a timely manner (e.g., meeting with a friend at $5 \mathrm{pm}$ ), perhaps increasing these tasks' ecological validity. In addition to using single-trial PM tasks, others have more generally increased the naturalistic features of their paradigms, such as Henry and colleagues (2014), who used the Virtual Week game that attempts to replicate the demands of daily life and also measured daily issues with PM via parent reports. Similarly, Talbot and Kerns (2014) measured parent reports of children's PM. Both studies found a positive association between PM in the task and performance on PM tasks in daily life. These findings are encouraging because they suggest that researchers are doing a good job of capturing PM processes in the laboratory that approximate PM success or failure in daily life.

Several articles is this special issue examine the role of reminder cues in PM performance (Causey \& Bjorklund, 2014; Kvavilashvili \& Ford, 2014; Walsh et al., 2014). After children initially failed to per- 
form the PM action, many studies have attempted to examine how many reminder cues children needed before they remembered the PM task. This is an important aspect of PM given that parents and other adults may give several scaffolding cues to support children's remembering of their intended actions. In these studies where reminder cues were offered, children were given full credit for a spontaneous PM response and a decreasing amount of credit as more specific cues were provided. We believe that these articles bring up two important issues. The first is the need for a consistent way to provide cues for children, which would result in greater comparability across research that uses reminders to see whether children can remember to perform the intention. The second issue is consideration of whether these cues are still measuring PM or at some point become measures of retrospective memory. As the reminder cues become more specific, many researchers ask children to recall what they needed to do in the PM task, which is a measure of retrospective memory rather than PM. For example, whereas the question "Was there something you forgot?" may still be measuring prospective processes, by the time children are asked "What did you need to do at the end of the game today?" it may be that this question is simply tapping into retrospective memory for what they were supposed to do. Future work in this area would do well to develop a consistent cueing scheme that could be used to ensure agreement on whether prospective or retrospective memory is being triggered by each successive reminder cue.

Several articles 2014 examine executive functions, and some examine whether they are mechanisms of PM development. In fact, more than half of the articles either directly or indirectly address the issue. Although findings are somewhat mixed, the overall picture is that executive control is an important factor driving the development of PM across childhood and adolescence. More controversial is what exact type of executive control process is responsible for developmental increases in PM. During the preschool years, inhibition fully mediates the relation between age and PM, suggesting that it is a strong candidate for a developmental mechanism (Mahy et al., 2014). Similarly, in 3- to 5-yearolds, a composite measure of executive control was related to high-incentive PM tasks in which children needed to get candy or remind the experimenter to give them candy at the end of the session (Causey \& Bjorklund, 2014). However, executive processes did not predict performance on a lowincentive PM task, suggesting that executive processes may be more important on certain types of PM tasks in line with the predictions of the multiprocess framework (McDaniel \& Einstein, 2000). During adolescence, task switching emerged as an important predictor of adolescents' PM alongside theory of mind ability (Altgassen et al., 2014), and caregiver reports of executive functions accounted for a significant amount of variance in PM in adolescents with prenatal alcohol exposure as well as typically developing adolescents (Robey et al., 2014), although there were inconsistent relations between PM and executive control during middle childhood in both typically and atypically developing children with autism spectrum disorders (Henry et al., 2014). Given that an important feature of PM tasks is the need to switch between the ongoing and PM tasks, inhibit the irrelevant responses, and keep in mind the rules of the task and what one is supposed to do, executive functions seem critical to PM. The next step in this area of research will be to tease apart the roles of the various components of executive functions and perhaps examine which processes of the PM task rely most on the various components of working memory, inhibition, set shifting, planning, and monitoring. The articles in this special issue establish executive control as a critical predictor of PM and suggest a role for at least some executive processes in the development of PM during childhood.

A long-standing issue in both the adult and child PM literatures is the relative independence or dependence of PM and retrospective memory processes. Given the adult literature, it is clear that PM relies on additional neural regions compared with retrospective memory (West \& Krompinger, 2005) and is often dissociable from retrospective memory processes in terms of behavioral performance. Early work on children showed that PM and retrospective memory seemed to be related in 3 -year-olds but were independent by the time children reached 5 years of age (Guajardo \& Best, 2000). Guajardo and Best (2000) suggested that these processes might be related early in development and then become dissociated later during the preschool years. The articles in the current issue address this debate and suggest that PM and retrospective memory are separable and distinct processes, at least during early childhood. Nigro and colleagues (2014) and Walsh and colleagues (2014) both find no relation between PM and retrospective memory processes in their tasks with preschoolers. Whereas Nigro and colleagues asked children to remind the experimenter to give a cellphone to a col- 
league for the PM task and asked them to recall what they needed to do for the retrospective memory task, Walsh and colleagues asked children to remember to stop at a given store (PM task) and then to remember what they were supposed to buy there (retrospective memory task). Although these tasks are diverse in their demands on PM and retrospective memory, they support the same conclusion that these processes are independent during early childhood. Kvavilashvili and Ford's (2014) results further support this finding by showing that although children are very accurate in predicting whether they will remember to carry out a PM task, their accuracy is poor when predicting whether they will remember something from the past (retrospective memory). If these two processes were related, one would expect that metamemory predictions would be similarly accurate or inaccurate for both types of memory. A large-scale longitudinal study examining these two types of memory processes independently across childhood (beyond the preschool years) is still needed to examine developmental changes in the relation between these two memory abilities; however, the current special issue supports the stance that these processes are distinct during early childhood.

On a theoretical note, several articles 2014 link research on children with adult theories of PM (e.g., multiprocess framework: McDaniel \& Einstein, 2000; PAM [preparatory attentional and memory processes] model: Smith, 2003; Smith \& Bayen, 2004) and even attempt to test the models of PM in children, a much needed step toward a more cohesive PM literature across the lifespan. For example, Leigh and Marcovitch (2014) show in 4- to 6-year-olds that controlled processes are recruited for the PM task and that relying on such processes results in slower ongoing task performance. This is important because it indicates that even a simple PM task that resulted in very high levels of performance is still associated with a cost to ongoing task performance in very young children. Further, other articles in the current issue examine ongoing task absorption or difficulty and cue salience (Henry et al., 2014; Mahy et al., 2014), which are factors predicted by the multiprocess framework to influence whether automatic and controlled processes are necessary (Einstein et al., 2005; McDaniel \& Einstein, 2000). In most cases, findings are generally supportive of the multiprocess framework that increasing cue salience and decreasing ongoing task difficulty or absorption should aid PM. Furthermore, Causey and Bjorklund (2014) show that individual differences in executive functions were related to high-interest PM task performance but not to low-interest PM task performance, suggesting that executive processes are more or less involved depending on task conditions, a potential extension of the multiprocess framework to the area of affective and motivational processes. These models of PM and their predictions for the role of controlled and automatic processes could be further informed by developmental data.

Finally, these articles suggest some promising directions for future work, including the role of metamemory in PM, goal maintenance and construction of event sequences as precursors to PM, the neural basis of PM in special populations, and the exact nature of the relation between PM and theory of mind across childhood. Metamemory and its relation with PM is relatively unexplored. Kvavilashvili and Ford (2014) show that children have much more accurate impressions of their PM ability than their retrospective memory ability, perhaps because children may receive more feedback regarding PM or have more experience in witnessing others or themselves failing PM tasks more frequently than retrospective memory tasks. Still, it is unknown whether children's knowledge of PM strategies relates to their use of strategies and, importantly, whether the use of such strategies actually improves PM performance. The examination of potential precursors to PM such as goal maintenance and being able to reproduce an event sequence accurately (Prabhakar \& Hudson, 2014) is important in understanding possible reasons why young children fail PM tasks. In three experiments, Prabhakar and Hudson (2014) show that 3-year-olds have difficulty in representing two sequential goals and that this failure is not due to difficulty with making inferences. As we study younger children, PM failures may be due to limitations in working memory or goal representation rather than to any executive difficulty associated with carrying out the action (see Leigh \& Marcovitch, 2014). Robey and colleagues (2014) examine relations between neural structure and PM in adolescents who were typically developing and those who were exposed to drugs prenatally. This represents one of the first links between examining the neural basis of PM during adolescence (but see Zöllig et al., 2007). Further work is needed to examine the neural structures associated with successful (and unsuccessful) PM in younger children because this would shed light on developmental processes involved in successful PM performance. Two studies in this special issue highlight the relation 
between theory of mind and PM-both citing the similarities in the two tasks in needing to consider something from a different perspective (either self-other or current self vs. later self). Ford and colleagues (2012) suggested that the self-projection hypothesis could explain why theory of mind is a good predictor of PM. Children who are able to project themselves into others' shoes or into the future should have an advantage in theory of mind or PM tasks, respectively. Self-projection may be a useful strategy to support PM given that work with adults has demonstrated the benefit of implementation intentions on PM (Chasteen, Park, \& Schwarz, 2001; Gollwitzer \& Brandstätter, 1997; McDaniel, Howard, \& Butler, 2008), which may rely on future simulation processes.

It is our hope that this special issue will fill some of the gaps in the PM literature and will also inspire new directions of research for the development of PM during childhood. This is still an open field that intersects with many other areas of development such as retrospective memory, executive control, theory of mind, and episodic future thinking and also has implications for children's daily functioning. PM is a critical skill for children to master in order to become independent, well-functioning adults. We hope that this issue inspires interest in the topic and also provides a thorough description of the development of PM across childhood, mechanisms of PM development, behavioral correlates of PM, and PM performance in special populations of children.

\section{References}

Altgassen, M., Schmitz-Hübsch, M., \& Kliegel, M. (2010). Event-based prospective memory performance in autism spectrum disorder. Journal of Neurodevelopmental Disorders, 2, 2-8.

Altgassen, M., Vetter, N. C., Phillips, L. H., Akgun, C., \& Kliegel, M. (2014). Theory of mind and switching predict prospective memory performance in adolescents. Journal of Experimental Child Psychology, 127, 163-175.

Altgassen, M., Williams, T. I., Bölte, S., \& Kliegel, M. (2009). Time-based prospective memory in children with autism spectrum disorder. Brain Impairment, 10, 52-58.

Atance, C. M., \& Jackson, L. K. (2009). The development and coherence of future-oriented behaviors during the preschool years. Journal of Experimental Child Psychology, 102, 379-391.

Brandimonte, M. A., Filippello, P., Coluccia, E., Altgassen, M., \& Kliegel, M. (2011). To do or not to do? Prospective memory versus response inhibition in autism spectrum disorder and attention-deficit/hyperactivity disorder. Memory, 19, 56-66.

Causey, K. B., \& Bjorklund, D. F. (2014). Prospective memory in preschool children: Influences of agency, incentive, and underlying cognitive mechanisms. Journal of Experimental Child Psychology, 127, 36-51.

Ceci, S. J., \& Bronfenbrenner, U. (1985). "Don't forget to take the cupcakes out of the oven": Prospective memory, strategic timemonitoring, and context. Child Development, 56, 152-164.

Chasteen, A. L., Park, D. C., \& Schwarz, N. (2001). Implementation intentions and facilitation of prospective memory. Psychological Science, 12, 457-461.

Einstein, G. O., \& McDaniel, M. A. (1990). Normal aging and prospective memory. Journal of Experimental Psychology: Learning, Memory, and Cognition, 16, 717-726.

Einstein, G. O., McDaniel, M. A., Thomas, R., Mayfield, S., Shank, H., Morrisette, N., et al (2005). Multiple processes in prospective memory retrieval: Factors determining monitoring versus spontaneous retrieval. Journal of Experimental Psychology: General, 134, 327-342.

Ford, R. M., Driscoll, T., Shum, D., \& Macaulay, C. E. (2012). Executive and theory-of-mind contributions to event-based prospective memory in children: Exploring the self-projection hypothesis. Journal of Experimental Child Psychology, 111, 468-489.

Gollwitzer, P. M., \& Brandstätter, V. (1997). Implementation intentions and effective goal pursuit. Journal of Personality and Social Psychology, 73, 186-199.

Guajardo, N. R., \& Best, D. L. (2000). Do preschoolers remember what to do? Incentive and external cues in prospective memory. Cognitive Development, 15, 75-97.

Henry, J. D., Terrett, G., Altgassen, M., Raponi-Saunders, S., Ballhausen, N., Schnitzspahn, K. M., et al (2014). A "Virtual Week" study of prospective memory function in autism spectrum disorders. Journal of Experimental Child Psychology, 127, 110-125.

Kerns, K. A. (2000). The CyberCruiser: An investigation of development of prospective memory in children. Journal of the International Neuropsychological Society, 6, 62-70.

Kerns, K. A., \& Price, K. J. (2001). An investigation of prospective memory in children with ADHD. Child Neuropsychology, 7 , $162-171$.

Kliegel, M., Mahy, C. E. V., Voigt, B., Henry, J. D., Rendell, P. G., \& Aberle, I. (2013). The development of prospective memory in young school children: The impact of ongoing task absorption, cue salience, and cue centrality. Journal of Experimental Child Psychology, 116, 792-810.

Kliegel, M., Ropeter, A., \& Mackinlay, R. (2006). Complex prospective memory in children with ADHD. Child Neuropsychology, 12, 407-419.

Kvavilashvili, L., \& Ford, R. M. (2014). Metamemory prediction accuracy for simple prospective and retrospective memory tasks in 5-year-old children. Journal of Experimental Child Psychology, 127, 65-81.

Kvavilashvili, L., Kyle, F., \& Messer, D. J. (2008). The development of prospective memory in children: Methodological issues, empirical findings, and future directions. In M. Kliegel, M. A. McDaniel, \& G. O. Einstein (Eds.), Prospective memory: Cognitive, neuroscience, developmental, and applied perspectives (pp. 115-140). Hove, UK: Psychology Press. 
Kvavilashvili, L., Messer, D. J., \& Ebdon, P. (2001). Prospective memory in children: The effects of age and task interruption. Developmental Psychology, 37, 418-430.

Leigh, J., \& Marcovitch, S. (2014). The cognitive cost of event-based prospective memory in children. Journal of Experimental Child Psychology, 127, 24-35.

Mackinlay, R. J., Kliegel, M., \& Mäntylä, T. (2009). Predictors of time-based prospective memory in children. Journal of Experimental Child Psychology, 102, 251-264.

Mahy, C. E., \& Moses, L. J. (2011). Executive functioning and prospective memory in young children. Cognitive Development, 26, 269-281.

Mahy, C. E. V., Moses, L. J., \& Kliegel, M. (2014). The impact of age, ongoing task difficulty, and cue salience on preschoolers' prospective memory performance: The role of executive function. Journal of Experimental Child Psychology, 127, 52-64.

McDaniel, M. A., \& Einstein, G. O. (2000). Strategic and automatic processes in prospective memory retrieval: A multiprocess framework. Applied Cognitive Psychology, 14, S127-S144.

McDaniel, M. A., Howard, D. C., \& Butler, K. M. (2008). Implementation intentions facilitate prospective memory under high attention demands. Memory \& Cognition, 36, 716-724.

Nigro, G., Brandimonte, M., Cicogna, P. C., \& Cosenza, M. (2014). Episodic future thinking as a predictor of children's prospective memory. Journal of Experimental Child Psychology, 127, 82-94.

Prabhakar, J., \& Hudson, J. A. (2014). The development of future thinking: Young children's ability to construct event sequences to achieve future goals. Journal of Experimental Child Psychology, 127, 95-109.

Robey, A., Buckingham-Howes, S., Salmeron, B., Black, M. M., \& Riggins, T. (2014). Relations among prospective memory, cognitive abilities, and brain structure in adolescents who vary in prenatal drug exposure. Journal of Experimental Child Psychology, 127, 144-162.

Shum, D., Cross, B., Ford, R., \& Ownsworth, T. (2008). A developmental investigation of prospective memory: Effects of interruption. Child Neuropsychology, 14, 547-561.

Smith, R. E. (2003). The cost of remembering to remember in event-based prospective memory: Investigating the capacity demands of delayed intention performance. Journal of Experimental Psychology: Learning, Memory, and Cognition, 29, 347-361.

Smith, R. E., \& Bayen, U. J. (2004). A multinomial model of event-based prospective memory. Journal of Experimental Psychology: Learning, Memory, and Cognition, 30, 756-777.

Talbot, K. D., \& Kerns, K. (2014). Event and time triggered remembering: The impact of ADHD on prospective memory performance in children. Journal of Experimental Child Psychology, 127, 126-143.

Voigt, B., Aberle, I., Schonfeld, J., \& Kliegel, M. (2011). Time-based prospective memory in schoolchildren. Zeitschrift für Psychologie, 219, 92-99.

Walsh, S. J., Martin, G. M., \& Courage, M. L. (2014). The development of prospective memory in preschool children using naturalistic tasks. Journal of Experimental Child Psychology, 127, 8-23.

Wang, L., Kliegel, M., Yang, Z., \& Liu, W. (2006). Prospective memory performance across adolescence. Journal of Genetic Psychology, 167, 179-188.

West, R., \& Krompinger, J. (2005). Neural correlates of prospective and retrospective memory. Neuropsychologia, 43, 418-433.

Williams, D., Boucher, J., Lind, S., \& Jarrold, C. (2012). Time-based and event-based prospective memory in autism spectrum disorder: The roles of executive function and theory of mind, and time-estimation. Journal of Autism and Developmental Disorders, 43, 1555-1567.

Zöllig, J., West, R., Martin, M., Altgassen, M., Lemke, U., \& Kliegel, M. (2007). Neural correlates of prospective memory across the lifespan. Neuropsychologia, 45, 3299-3314.

Caitlin E.V. Mahy
Department of Psychology, University of Oregon,
Eugene, OR 97403, USA

Department of Psychology, University of Geneva, 1205 Geneva, Switzerland

E-mail address: cmahy@uoregon.edu

Matthias Kliegel

Department of Psychology, University of Geneva, 1205 Geneva, Switzerland

Stuart Marcovitch Department of Psychology, University of North Carolina - Greensboro, Greensboro, NC 27402, USA 